Z. klin. Cham. u. klin. Biochem.

7. Jg., S. 194-196, März 1969

\title{
Mengenabhängigkeiten bei der Papierchromatographie von Aldosteron
}

\author{
Von W. Schimidimand, L. Reschike und G. H. Leistner \\ Aus der Mediziniscben Universitäts-Klinik Bonn-Venusberg (Direktor: Prof. Dr. A. Heymer)
}

(Eingegangen am 5. Dezember 1968)

Es wird über Beobachtungen bei der Papierchromatographic von Aldosteron- $\left[1,2-{ }^{3} \mathrm{H}\right](0,05 \mu \mathrm{g} ; 1 \mu \mathrm{C})$ unter Zusatz steigender Mengen von inaktivem Aldostcron $(0,1,5,10,20,40 \mu \mathrm{g})$ berichtet: Mit steigendem Gehalt an inaktivem Aldosteron nimmt die adsorbierte $\mathrm{Menge}$ von Aldosteron- $\left[1,2{ }^{3} \mathrm{H}\right]$ an der Auftragsstelle ab. Die Gesamtmenge an adsorbiertem Aldosteron liegt dabei unter $5 \mu \mathrm{g}$. - Bei Substanzmengen ron mehr als $10 \mu \mathrm{g}$ inaktivem Aldosteron werden die Aldosteronpeaks auf den Radiopapierchromatogrammen trotz des Nachweises eines konstanten Gehalts an Aldosteron- $\left[1,2-{ }^{3} \mathrm{H}\right]$ kleiner. Die Größe dieser Peaks ermöglicht daher keinen zwingenden Schluß auf dic Menge an radioaktiv markierter Substanz. - Eine Näherungsrechnung gibt einen Anhalt für die auf der gesamten Laufstrecke adsorptiv gebundene Aldosteronmenge. Diese muß bei der Auswertung berücksichtigt werden.

\section{The effect of loading in the paper chromatograpby of aldosterone}

Observations are reported on the paper chromatography of $\left[1.2-{ }^{3} \mathrm{H}\right]$-aldosterone $(0.05 \mu \mathrm{g} ; 1 \mu \mathrm{C})$ with the addition of increasing amounts of unlabelled aldosterone $(0,1,5,10,20,40 \mu \mathrm{g})$. As the amount of unlabelled aldosterone was increased, the amount of [1.2- $\left.{ }^{3} \mathrm{H}\right]-\mathrm{aldosterone}$ adsorbed at the starting point decreased. The total quantity of adsorbed aldosterone was less than $5 \mu \mathrm{g}$. With quantities of unlabelled aldosterone higher than $10 \mu \mathrm{g}$, the aldosterone peak on the radio-paper chromatograms was smaller, despite the demonstration of a constant level of $\left[1.2-{ }^{3} \mathrm{H}\right]$-aldosterone. The height of this peaks therefore does not permit a firm conclusion as to the quantity of radioactive material. An approximate calculation indicates that a quantity of aldosterone is adsorbed over the whole chromatographic pathway. This must be allowed for in the final analysis.

Im Rahmen von Untersuchungen über die Bestimmung des Aldosterongehalts von Urinen mit der Doppelisotopen-Verdünnungsanalyse (1) wurden vergleichsweise auch reine Aldosteronlösungen herangezogen, die durch Zugabe steigender Mengen von inaktivem Aldosteron zu gleichbleibenden. Mengen von tritiiertem Aldosteron in Äthylalkohol hergestellt wurden. Die aus diesen Lösungen gewönnenen Aldosteronproben (s. Methodik) wurden der gleichen papierchromatographischen Behandlung wie aus Urinextrakten hergestellte Urinproben unterworfen.

Durch Vergleich des papierchromatographischen Verhaltens des tritiierten Aldosterons in Abhängigkeit von der Menge an zugesetztem inaktiven Aldosteron ist ein Einfluß auf die Menge des an der Auftragssstelle adsorptiv gebundenen tritiierten Aldosterons sowie auf die Höhe radiopapierchromatographisch aufgenommener Peaks nach Wanderung der Substanz festzustellen. Auf Grund der Befunde wird durch Näherungsrechnung die auf der gesamten Laufstrecke adsorptiv gebundene Aldosteronmenge bestimmt.

\section{Methodik}

Für die Versuche wurde Aldosteron- $\left.\left[1,2-{ }^{3} \mathrm{H}\right]^{1}\right)$ verwendet, dessen spezifische Aktivität $7,8 \mathrm{C} / \mathrm{mM}$ ol betrug. $0,1 \mathrm{mC}$ Aldosteron$\left[1,2-{ }^{3} \mathrm{H}\right]$ wurden mit Äthanol auf $50 \mathrm{ml}$ aufgefüllt. Je $0,5 \mathrm{ml}$ $(=0,05 \mu \mathrm{g} ; 1 \mu \mathrm{C})$ wurden entnommen und mit steigenden Zusätzen $(1,5,10,20,40 \mu \mathrm{g})$ an inaktivem Aldosteron versetzt. Die Aldosteronproben wurden durch Eindampfen dieser Lösungen und Aufnahme des Rückstandes in Methanol/Chloroform 1) Hersteller: RADIOCHEMICAL CENTRE, Amersham,
Buckinghamshire/England.
$(1: 1 \mathrm{v} / \mathrm{v})$ gewonnen. Die Urinproben wurden durch Extrahieren von'angesäuertem Urin mit Chloroform, Eindampfen des Urinextrakts und Aufnehmen des Rückstandes mit Methanol/Chloroform $(1: 1 \mathrm{v} / \mathrm{v})$ hergestellt.

Für die Chromatographie wurden sechs je $2 \mathrm{~cm}$ breite Papierchromatographiestreifen (SCHLEICHER \& SCHÜLx, 2043 b Mgl) verwendet, die aus einem Blatt von $14,5 \mathrm{~cm}$ Breite und $54 \mathrm{~cm}$ Höhe so gefertigt waren, daß sie oben in einer Höhe von $4 \mathrm{~cm}$ und unten in einer Höhe von $9 \mathrm{~cm}$ zusammenhingen und im übrigen einen gegenseitigen Abstand von $0,5 \mathrm{~cm}$ aufwiesen.

Nach dem Auftragen der Proben auf dic Chromatographiestreifen wurde nach NeHER (2) $24 \mathrm{Stdn}$. bei $20-22^{\circ}$ absteigend im System Wasser/Methanol $(1: 1 \mathrm{v} / \mathrm{v})$ als stationäre Phase und Benzol/Petroläther $(1: 1 \mathrm{v} / \mathrm{v})$ als mobile Phase chromatographicrt. Die beiden Phasen waren vorher gegenseitig gesättigt. Vor dem Einbringen in die Chromatographietanks wurden die Streifen mit der stationären Phase bis $1 \mathrm{~cm}$ vor der A:tftragsstelle imprägniert. Die Chromatographietanks waren zur Sättigung der Atmosphäre mit den verwendeten Lösungsmitteln innen mit Filtricrpapieren ausgekleidet, die aber vorn nicht ganz überlappten und ein etwa $4 \mathrm{~cm}$ breites Sichtfenster freiließen. Parallel-Proben wurden auf zwei Blätter (A und $B$ ) aufgetragen.

Die entwickelten Chromatogramme wurden radiopapierchromatographisch ${ }^{2}$ a asgewertet. Von den Chromatographiestreifen wurden Kontaktaufnahmen im UV-Licht $(\lambda=254 \mathrm{~nm})$ angcfertigt.

Die Radiopapierchromatogramme in Abbildung 1 sind in der Länge im Maßstab 1:5, in der Höhe im Maßstab 1:3 dargestellt.

An Hand der radiopapicrchromatographischen Peaks wurde dic Substanz aus den entsprechenden Chromatographieabschnitten mit Methanol eluiert. Es wurden jedoch für die Eluierung die unmittelbar an den Auftragssstellen liegenden Aktivitătspeaks nicht berücksichtigt. Die gewonnenen Eluate wurden im Flüssigkeitsszintillationszähler ${ }^{3}$ ) gemessen.

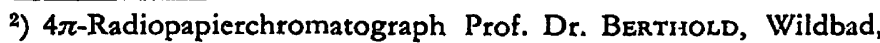
mit Strahlenmeßgerät FH 49, Frieseke \& Hoepfner, Eriangen. 3) TRI-CARB Modell 3002, Packard Instrument CO., La Grange, Ill. USA. 


\section{Ergebnisse}

1. Die Radioaktivität an der Auftragsstelle Ein Vergleich der Radiopapierchromatogramme (Abb. 1) ergibt, daß bei den lediglich $0,05 \mu \mathrm{g}$ Aldosteron$\left[1,2-{ }^{3} \mathrm{H}\right]$ enthaltenden Proben (I) der größere Teil der Aktivität an der Auftragsstelle verblieben und nur der

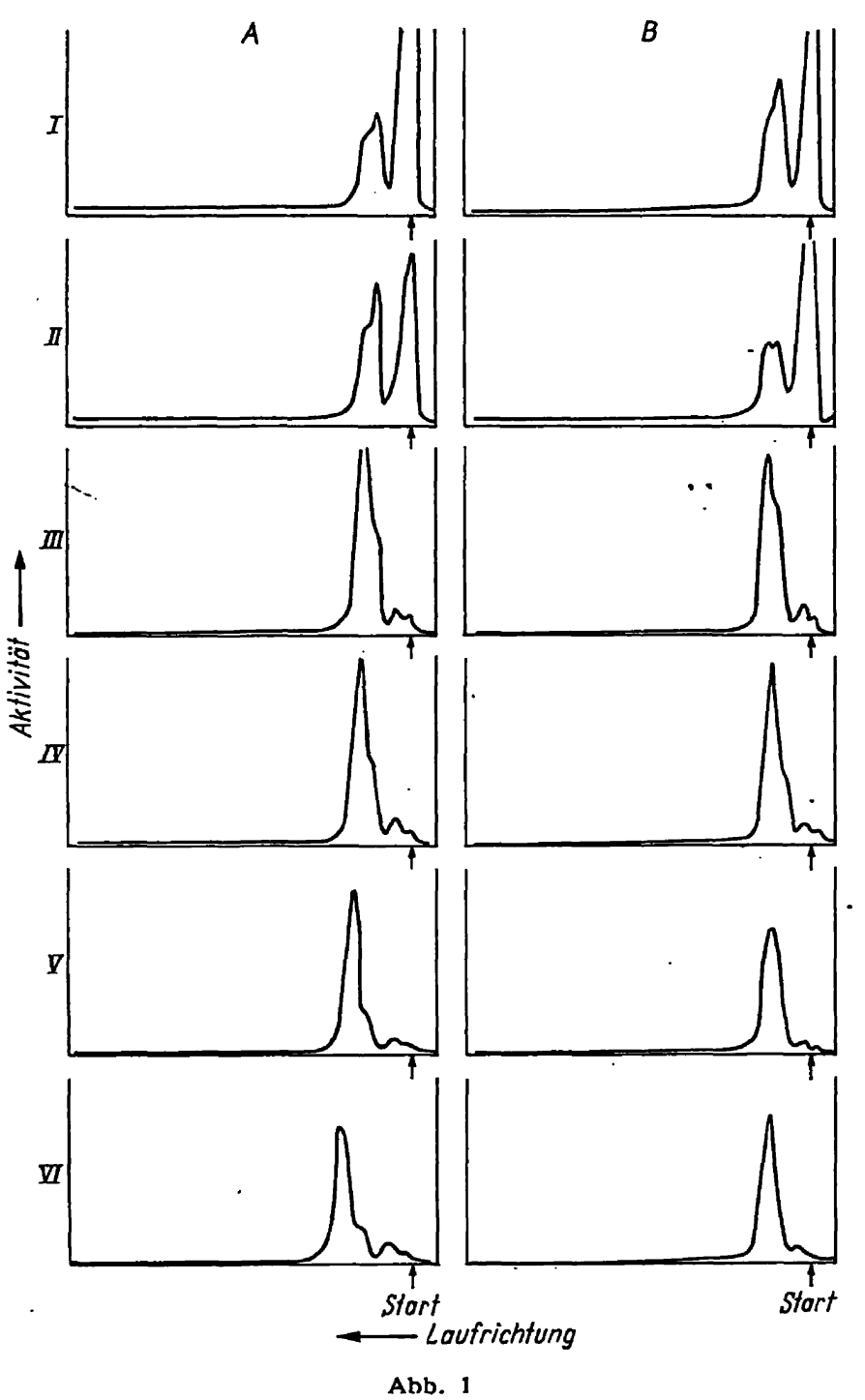

Radiopapierchromatogramme: -GTeichbleibende Aldosteron-[1,2-:H]Aktivität ( $1 \mu \mathrm{C} ; 0,05 \mu \mathrm{g})$ und steigende Zusätze von inaktivem Aldosteron: I ohne Zusatz, II $1 \mu \mathrm{g}$, III $5 \mu \mathrm{g}$, IV $10 \mu \mathrm{g}, \mathrm{V} 20 \mu \mathrm{g}$, VI $40 \mu \mathrm{g}$.
Senkrecht nebeneinander Ergebnis zweier Parallel-Läufe A und B

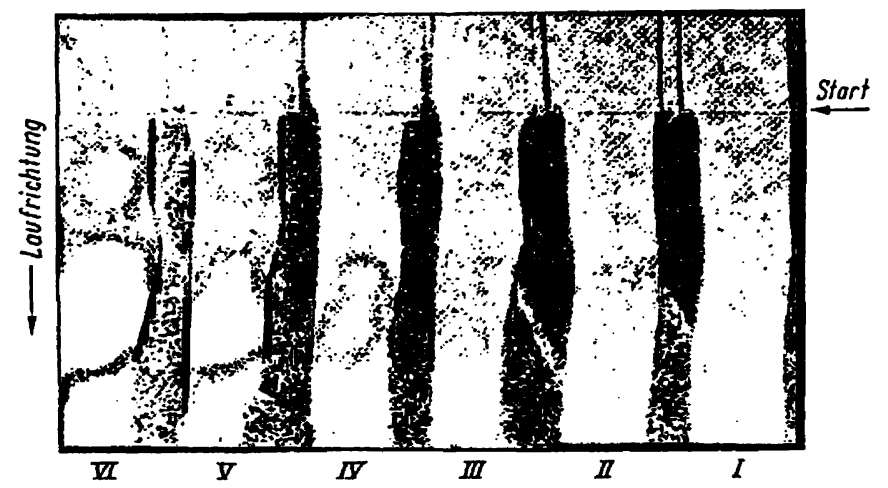

Abb. 2

UV-Kontaktaufnahme der Chromatogramme von $0,05 \mu \mathrm{g}=1 \mu \mathrm{C}$ Aldosteron-[1,2-'H] (Lauf B, oberer Teil) Von rechts nach links steigende 2 salze von inaktivem Aldosteron: I ohne Zusatz, II I $\mu \mathrm{g}$, III $5 \mu \mathrm{g}$, IV $10 \mu \mathrm{g}$, V $20 \mu \mathrm{g}$, vi $40 \mu \mathrm{g}$ kleinere Teil gewandert ist. Die Radiopapierchromatogramme mit einem Zusatz von $1 \mu \mathrm{g}$ inaktivem Aldosteron (II) zeigen (deutlich für die Probe $\mathrm{Al}$ ) eine $\mathrm{Ab}$ nahme der Radioaktivität an der Auftragsstelle unter stärkerer Ausbildung des davor liegenden Peaks.

Mit steigendem Zusatz an inaktivem Aldosteron wird die Radioaktivitãt an der Auftragsstelle geringer, während. der durch Wanderung der Substanz entstandene Peak deutlicher in Erscheinung tritt.

Diese Beobachtungen lassen darauf schließen, daß sehr geringe Mengen Aldosteron, wie sie in den Proben I A und I B vorliegen, zum großen Teil durch Adsorption an den Auftragsstệlen verbleiben. Die bei steigendem Zusatz von inaktivem Aldosteron dort eintretende Abnahme an Radioaktivität kann dadurch erklärt werden, daß die adsorptionsfähigen Stellen zunehmend durch inaktives Aldosteron blockiert werden, so $\mathrm{daB}$ immer weniger Aldosteron- $\left[1,2-{ }^{3} \mathrm{H}\right]$ an der Auftragsstelle liegen bleibt. Die Radiopapierchromatogramme lassen den Schluß zu, daß die an der Auftragsstelle adsorbierte Menge weniger als $0,05 \mu \mathrm{g}$ beträgt, da selbst beim Auftragen dieser Menge allein ein kleinerer Teil des Aldosterons der Verteilungschromatographie unterliegt (s. auch unter 2., Ergebnisse der Flüssigkeitsszintillationsmessungen).

Kontaktphotographisch läßt sich über die Menge des an der Auftragsstelle verbliebenen inaktiven Aldosterons sagen, daß es sich um weniger als $5 \mu \mathrm{g}$ handeln muß, da Mengen von $5 \mu \mathrm{g}$ eben noch nachweisbar sind (vgl. in Abb. 2 die Chromatogramme I bzw. II mit III). Selbst bei den Proben VI sind an den Auftragsstellen weniger als $5 \mu \mathrm{g}$ Aldosteron verblieben.

Urinproben, die nach der Aufarbeitung und vor dem Auftragen auf Papierchromatographiestreifen mit $0,5 \mathrm{ml}$ Aldosteron-[1,2- $\left.{ }^{3} \mathrm{H}\right](0,05 \mu \mathrm{g} ; 1 \mu \mathrm{C})$ versetzt worden sind, zeigen an der Auftragsstelle ebenfalls kaum Radioaktivität, wie aus dem beispielsweise beigefügten Radiopapierchromatogramm (Abb. 3) ersichtlich ist.

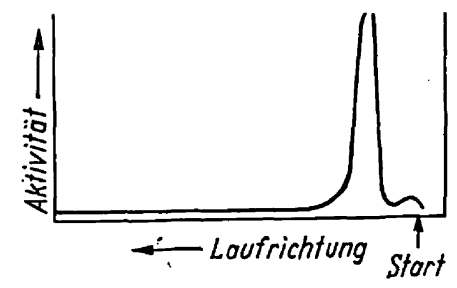

Abb. 3

Radiopapierchromatogramm einer Urinprobe, chromatographiert nach Zusatz von $0,05 \mu \mathrm{g}=1 \mu \mathrm{C}$ Aldosteron- $\left[1,2-{ }^{3} \mathrm{H}\right]$

Diese Phänomene lassen sich am einfachsten als eine Konkurrenz zwischen Adsorptions- und Verteilungschromatographie deuten: Kleinste Mengen von Aldosteron werden an der Auftragsstelle adsorbiert, während mit steigender Substanzmenge in zunehmendem Maße die Verteilungschromatographie in Erscheinung tritt. Bei den Urinproben können bei der Blockierung adsorptiver Stellen am Start außer inaktivem Aldosteron auch andere in den Urinen enthaltene Stoffe cine Rolle spielen. 
2. Sind Radiopapierchromatogramme von Aldosteron-[1,2- $\left.{ }^{3} \mathrm{H}\right]$-Proben quantitativ auswertbar?

Die Auswertung der Radiopapierchromatogramme (Abb. 1) ergibt außerdem, daß das von der Auftragsstelle fortgewanderte Aldosteron bei den Proben mit 5 und $10 \mu \mathrm{g}$ Aldosterongehalt (III und IV) den höchsten Peak aufweist, während dieser bei den Proben mit 20 bzw. $40 \mu \mathrm{g}$ Aldosteron (V und VI) wieder kleiner wird. Demgegenüber läßt die Kontaktaufnahme (Abb. 2) eine mit zunehmendem Aldosterongehalt der Proben deutlich stärker werdende Aufhellung im Bereich der radiopapierchromatographisch dargestellten Aldosteronpeaks erkennen.

Dieser Befund ist damit zu erklären, daß mit zunehmender Substanzmenge und abnehmender spezifischer Aktivität des Aldosterons die Selbstabsorption der weichen $\beta$-Strahlen des Tritiums größer wird. Diese Annahme wurde durch Flüssigkeitsszintillationsmessungen bestătigt. Die erhaltenen Werte sind in Abbildung 4 graphisch dargestellt. Wie zu ersehen ist, steigt die Radioaktivität mit zunehmendem Gehalt an inaktivem

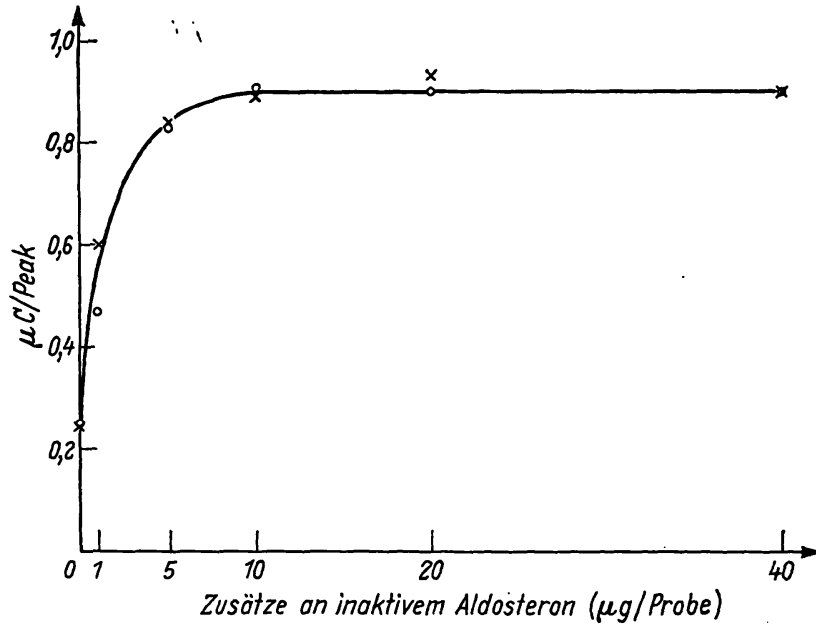
Abb. 4

Radioaktivität von papierchromatographisch gewandertem Aldosteron- $\left[1,2-^{3} \mathrm{H}\right]$ bei Proben von Aldosteron- $\left[1,2-{ }^{3} \mathrm{H}\right](0,05 \mu \mathrm{g}=1 \mu \mathrm{C})$ mit steigenden Zusätzen an inaktivem Aldosteron; Flüssigkeits$\mathbf{x}=$ Proben Lauf A $\quad 0=$ Proben Lauf $B$

Aldosteron und erreicht für die Proben IV, V und VI im Gegensatz zu den kleiner werdenden Peaks auf den Radiopapierchromatogrammen - einen praktisch konstanten Wert von $0,90 \mu \mathrm{C}$ (IV A: $0,89 \mu \mathrm{C}$; IV B $0,93 \mu \mathrm{C}$ ), woraus sich im übrigen ergibt, daß sich $9 / 10$ der eingesetzten Radioaktivität in diesen eluierten Peaks befand. Die durch diese Messungen im TRI-CARB erhaltenen Befunde verdeutlichen somit, daß die Größe der Peaks auf dem Radiopapierchromatogramm bei tritiiertem Aldosteron nur sehr bedingt Rückschlüsse auf dessen Menge erlaubt.
Die Auswertung der Flüssigkeitsszintillationsmessungen zur Bestimmung der Radioaktivität im Bereich der gewanderten Peaks ergibt weiterhin, daß die Proben III mit 0,84 bzw. $0,83 \mu \mathrm{C}$ den für die Proben IV, $\mathrm{V}$ und VI konstanten Wert von $0,90 \mu \mathrm{C}$ schon nahezu erreichen, während die Werte für die Proben II $(0,60$ bzw. $0,47 \mu \mathrm{C}$ ) und insbesondere für die Proben I A bzw. I B $(0,24$ bzw $0,25 \mu \mathrm{C})$ erheblich niedriger sind. Damit errechnet sich für die Proben I bzw. II bei einer eingesetzten Radioaktivität von $1 \mu \mathrm{C}$ ein Verbleib von etwa $75 \%$ bzw. $40-50 \%$ an den Auftragsstellen (vgl. die Ausführungen unter 1.).

3. Substanzverluste während der Papierchromatographie von Aldosteron

Auf Grund dieser Feststellung, daß von der Radioaktivität der Proben I A bzw. I B nur etwa 25\% gewandert sind, ist weiterhin zu folgern, daß der an der Auftragsstelle verbliebene Anteil von $75 \%$ ausreicht, um die dort vorhandenen adsorptionsfähigen Stellen durch Substanz abzusättigen. Wird die Größe der Auftragsstelle mit etwa $1 \mathrm{~cm}^{2}$ angenommen, so sind damit etwa $0,04 \mu \mathrm{g}$ Aldosteron pro $\mathrm{cm}^{2}$ zur Sättigung erforderlich.

Darüber hinaus ist neben einer die Wanderung der Substanz bedingenden Verteilungschromatographie aber auch noch eine Adsorption der Substanz längs der zurückgelegten Wanderungsstrecke in Betracht zu ziehen. Unter der Voraussetzung, daß sich auch die im Laufe des Analysenganges hergestellten Aldosteronderivate in den von NeHER angewandten Systemen bei der Besetzung adsorptiv wirksamer Stellen genau so verhalten wie Aldosteron selbst, würde sich beispielsweise bei den wenigstens vier Chromatogrammen nach NEHER mit einer gesamten Wanderungsstrecke des Aldosterons und seiner Derivate von 40 bis $50 \mathrm{~cm}$ ein Gesamtverlust von 3,2 bis $4 \mu \mathrm{g}$ adsobiertem Aldosteron auf der überwanderten Fläche von 80 bis $100 \mathrm{~cm}^{2}$ errechnen.

Bei der Chromatographie von Urinen sind dagegen, wie unter 1. erörtert wurde, die durch Adsorption bedingten Aldosteronverluste sehr gering. Eine auf einem Vergleich von Urinproben mit reinen Aldosteronproben bei gleichem Trennungsgang beruhende Bestimmung muß demzufolge für die Urine zu hohe Aldosteronwerte ergeben. Der Fehler fällt um so weniger ins $\mathrm{Ge}-$ wicht, je größer der für die Analyse verwendete aliquote Teil des Tagesurins und die dazu in Beziehung gesetzte Menge an reinem Aldosteron ist.

Wir danken Frau Barbara Kempree und Fräulein Gịsela Peters für ihre Mitarbeit bei den chromatographischen Arbeiten.

Der Deutschen Forschungsgemeinschaft danken wir für die Unterstützung der Arbeit.

\section{Literatur}

1. Kliman, B. und R. E. Peterson, J. biol. Chemistry 235, 1639 (1960). - 2. NeHER, R., 9. Symp. dtsch. Ges. Endokrinologie
Wiesbaden-Mainz 1962, S. 21-39, Springer Verlag, Berlin-Göttingen-Heidelberg (1963).
Dr. W. Schmidtmann, Dipl.-Chem. Medizinische Universitäts-Klinik 53 Bonn-Venusberg 